\title{
A high prevalence of low bone mineral density in children with glycogen storage disease type III
}

\section{Type}

Research paper

\section{Keywords}

osteoporosis, pediatrics, DXA scan, GSD III

\begin{abstract}
Introduction

Glycogen storage disease III (GSD III) is an inborn error of carbohydrate metabolism that involves mainly the liver and skeletal muscles with rare potential systemic complications as osteoporosis. The aim of work of the current study was to screen children with GSD III for osteoporosis using Dualemission-X-ray absorptiometry (DXA) scan and to measure associated parathormone (PTH) and vitamin $\mathrm{D}$. Our secondary objective is to correlate between the degree of osteoporosis and the muscle state as well as the metabolic control.
\end{abstract}

Material and methods

This cross sectional study included 25 GSD III pediatric cases. The liver biochemical profile, creatine kinase (CK), vitamin D2, PTH levels were tested in addition to electromyogram (EMG),

echocardiography and DXA. Twenty-five age and sex matched normal healthy controls were subjected to vitamin D2 analysis and compared to cases.

\section{Results}

The mean $\pm S D$ age of the patients was $10.52 \pm 3.1$ years ranging between $5-18$ years. Twenty-one patients $(84 \%)$ had elevated CK levels, 14 patients $(56 \%)$ had myopathy and three $(12 \%)$ had sensory polyneuropathy and almost half of the patients had a mild degree of cardiac muscle hypertrophy. Ten patients (40\%) had elevated PTH levels. Twenty-one patients (84\%) and $96 \%$ of the controls had vitamin D2 deficiency. Thirteen patients (52\%) had low BMD; two of them had osteoporosis. Patients with high CK levels significantly had low BMD $(P=0.04)$.

\section{Conclusions}

Pediatric GSD III patients have a significant prevalence of developing low BMD. It is strongly associated with myopathic changes but not significantly related to metabolic control. 


\begin{abstract}
:
Introduction: Glycogen storage disease III (GSD III) is an inborn error of carbohydrate metabolism that involves mainly the liver and skeletal muscles with rare potential systemic complications as osteoporosis. The aim of work of the current study was to screen children with GSD III for osteoporosis using Dual-emission-X-ray absorptiometry (DXA) scan and to measure associated parathormone (PTH) and vitamin D. Our secondary objective is to correlate between the degree of osteoporosis and the muscle state as well as the metabolic control. Material and methods: This cross sectional study included 25 GSD III pediatric cases. The liver biochemical profile, creatine kinase (CK), vitamin D2, PTH levels were tested in addition to electromyogram (EMG), echocardiography and DXA. Twenty-five age and sex matched normal healthy controls were subjected to vitamin D2 analysis and compared to cases. Results: The mean \pm SD age of the patients was $10.52 \pm 3.1$ years ranging between 5-18 years. Twenty-one patients $(84 \%)$ had elevated CK levels, 14 patients $(56 \%)$ had myopathy and three $(12 \%)$ had sensory polyneuropathy and almost half of the patients had a mild degree of cardiac muscle hypertrophy. Ten patients (40\%) had elevated PTH levels. Twenty-one patients
\end{abstract} (84\%) and $96 \%$ of the controls had vitamin D2 deficiency. Thirteen patients (52\%) had low BMD; two of them had osteoporosis. Patients with high CK levels significantly had low BMD ( $\mathrm{P}=0.04)$. Conclusion: Pediatric GSD III patients have a significant prevalence of developing low BMD. It is strongly associated with myopathic changes but not significantly related to metabolic control.

Key words: DXA scan; GSD III; pediatrics; osteoporosis 


\section{Introduction:}

Glycogen storage disease type III (GSD III) is an inborn error of carbohydrate metabolism caused by deficient activity of glycogen debranching enzyme. It is characterized by liver, cardiac and skeletal muscle involvement. The presence of systemic complications such as growth retardation, ovarian polycystosis, diabetes mellitus and osteopenia/osteoporosis has been reported. The pathogenesis of osteoporosis in these patients is still unclear [1].

Osteoporosis, literally "porous bone", is a disease characterized by weak bone with increased risk to bone fractures [2]. Osteoporosis can result in devastating physical, psychosocial, and economic consequences. It is often overlooked and undertreated, however, in large part because it is clinically silent before manifesting as fracture [3]. Early evaluation, screening, and treatment of bone disorders in patients with liver disease are essential to minimize fracture risk and to improve clinical outcome and quality of life [4].

Different hormones and proteins cooperate in determining bone mineral density (BMD). Bone status is directly controlled by parathyroid hormone (PTH), calcitonin, osteocalcin and vitamin D [1]. Dual-emission-X-ray absorptiometry (DXA) technique is considered the clinical gold standard to measure BMD [5].

The World Health Organization (WHO) defines osteoporosis in adults as BMD at least 2.5 standard deviations (SDs) below peak. Although this definition is functionally valid for adults, it is not appropriate for children as they have not yet attained peak bone mass. Instead, Z scores are used in children [6]. 
Both children and adults with GSD III may have significantly reduced BMD on DXA scanning, which is probably related to myopathic changes [7], abnormal metabolic environment and altered nutrition [1]. Although some studies reported a high incidence of bone fractures in GSD III patients [8], there is still paucity in the literature reporting these data in the younger age groups.

The aim of work of the present study is to screen children with GSD III for osteoporosis using DXA scans and to measure associated PTH and vitamin D2 levels. Our secondary objective is to correlate between the degree of osteoporosis and the muscle state as well as the metabolic control.

\section{Material and methods:}

This cross sectional study was carried out at the Pediatric Hepatology Unit, Cairo University Children's Hospital. The study included patients with established diagnosis of GSD III who were recruited during the period from November 2017 to July 2018. The study protocol was approved by the Institutional Review Board and the Research Ethics Committee of Kasr Alainy Medical School, Cairo University after taking an informed consent from the patients' parents.

Children between 5-18 years of age were included. Patients were excluded if they had any other associated chronic disease. GSD III was suspected clinically and the diagnosis was confirmed by performing a liver biopsy and measuring the amylo-1,6 glucosidase enzyme in erythrocytes $[\mathbf{9}, \mathbf{1 0}]$.

All patients were subjected to the following: 
1-History taking including: Epidemiological data, exercise habits, symptoms suggestive of hypoglycemia, myopathy, cardiac affection, pulmonary or systemic congestion, history of fractures, compliance on oral uncooked corn starch

2-Clinical examination: with special emphasis on abdominal, neurological and cardiac examination

\section{3-Investigations:}

Laboratory assessment: Liver function tests, serum random blood sugar (RBS), triglycerides, lactate and uric acid (to assess the metabolic control), creatine kinase (CK) for evidence of myopathy. Bone metabolism was studied by evaluating serum calcium, phosphorus, PTH and 25-hydroxy vitamin D (using Roche diagnostics). Vitamin D2 level was also assessed in 25 age and sex matched healthy controls. Vitamin D2 was considered normal if $>30 \mathrm{ng} / \mathrm{ml}$, insufficient if between $21-29 \mathrm{ng} / \mathrm{ml}$ and deficient if $<20$ $\mathrm{ng} / \mathrm{ml}[11]$.

\section{Radiological assessment:}

- Electromyography (EMG) and nerve conduction velocity (NCV) were done using micromed system (Molgiano Veneto-Italy).

- Echocardiographic evaluation was performed using the Vivid 5 and Vivid 6 echocardiographic machines (GE-Vingmed, Horten, Norway).

- Evaluation of BMD using DXA scan (GE, Lunar, Hologic 4500W, USA). The skeletal sites for BMD measurements in children were the lumbar spine and total body. $\mathrm{BMD}$ results were reported as areal $\mathrm{BMD}(\mathrm{g} / \mathrm{cm} 2)$ and Z-score. The Z-score was reevaluated, taking into account adjustment for bone age [12]. Volumetric density of the 
lumbar spine (aBMD) was also estimated mathematically in L2-L4 as proposed by Carter et al. [13]. Interpretation of DXA scan according to Bachrach and Gordon [14] was performed as follows:

- Osteoporosis: Z-score $\leq-2.0$ adjusted for age, gender, body size + significant fracture (Lower extremity long bone, vertebral compression or $\geq 2$ upper extremity long-bone fractures).

- When BMD Z-score is $\leq-2.0$ and no relevant fracture history is present, the term "low BMD (LBMD) for chronological age" was used

- Z-score between -1.0 and -2.0 is defined as the low range of normality.

\section{Statistical analysis:}

Data was collected, tabulated and were statistically described in terms of mean $\pm \mathrm{SD}$, median (Interquartile range [IQR)]), or frequencies and percentages when appropriate. Comparison of numerical variables between the study groups was done using Student $t$ test for normally distributed data and Mann Whitney $U$ test for non-normally distributed data. For comparing categorical data, Chi-square $\left(\chi^{2}\right)$ test was performed. Exact test was used instead when the expected frequency was less than 5. Correlation between various variables was done using Spearman rank correlation equation. $\mathrm{P}$ values less than 0.05 were considered statistically significant. All statistical calculations were done using computer program IBM SPSS (Statistical Package for the Social Science; IBM Corp, Armonk, NY, USA) release 22 for Microsoft Windows. 


\section{Results:}

The study included 25 patients with GSD III; 14 of them were males (56\%) with age ranging between 5 to 18 years. The clinical data of the included patients are summarized

\section{in Table 1.}

Laboratory investigations showed that most patients had elevated transaminases (96\%).

(Table 2). Regarding the parameters of metabolic control, 20\% of the patients had hypoglycemia, 50\% had elevated triglycerides levels and about one third had elevated serum lactate. There were no statistically significant correlation between RBS level and both triglycerides and lactate $(r=-0.120, p=0.567)$ and $(r=0.250, p=0.228)$ respectively. Twenty-one patients (84\%) had elevated CK level ranging between 70-2489 U/1 (Table 3). There was no statistically significant correlation between the age of cases and CK level $(r=0.117, p=0.579)$. Patients with high $\mathrm{CK}$ levels significantly had elevated alanine aminotransferase (ALT) levels than patients with normal CK levels $(\mathrm{P}=0.02)$. There was a statistically significant positive correlation between CK and both ALT and aspartate aminotransferase (AST) levels [ $(\mathrm{r}=0.676, \mathrm{p}=0.000)$ and $(\mathrm{r}=0.468, \mathrm{p}=0.018)$ respectively]. As regards results of EMG and NCV, 14 patients (56\%) had myopathy and three patients (12\%) had sensory polyneuropathy. Cases with myopathy significantly had higher CK levels than cases without myopathy $(\mathrm{p}=0.001)$. All patients with myopathy had elevated CK level. About 35\% of the patients with myopathy were not adherent to oral starch therapy. Four out of the 6 patients who experienced manifestations suggestive of muscle weakness, had myopathic changes in EMG and 3 had cardiomyopathy. Half of the patients had a mild degree of cardiac muscle hypertrophy by echocardiography, out of them 11 patients (44\%) had concentric left ventricular 
hypertrophy (LVH) and 3 patients (12\%) had septal hypertrophy. Although $86 \%$ of the patients with cardiac hypertrophy had elevated CK, its level was comparable between patients with and without cardiac muscle hypertrophy.

Investigations of bone metabolism (Table 3 ) showed that 13 patients (52\%) had hypocalcemia, 4 (16\%) had low phosphorus levels, one patient (4\%) had elevated alkaline phosphatase (ALP) and ten patients (40\%) had elevated PTH levels. There was no correlation between PTH and both calcium and phosphorus levels $[(\mathrm{r}=-0.167, \mathrm{p}=0.426),(\mathrm{r}=0.089, \mathrm{p}=0.672)$ respectively]. In order to assess the validity of vitamin D2 measurements, vitamin D2 level was also assessed in 25 healthy controls. Cases and controls were age and sex matched. Vitamin D2 was decreased in the 2 groups without a statistically significant difference [Median (IQR) in cases and controls: $10.8(8.1)$ and $8.8(5.5) \mathrm{ng} / \mathrm{ml}$ respectively, $\mathrm{P}=0.68]$. Twenty-one patients (84\%) and 96\% of the controls had vitamin D2 deficiency. Two patients (8\%) had vitamin D2 insufficiency. The last two patients who had normal vitamin D2 were already on vitamin D supplementation.

As regards the result of DXA scan in the study group, 13 patients (52\%) had LBMD, two of them met the definition of osteoporosis (history of long bone fractures three times in the first patient and two times in second patient plus LBMD). Five patients (20\%) had LBMD at spine only, one patient (4\%) had low total density and 7 patients (28\%) had LBMD at both sites. Median (IQR) of density at the spine was -1.90 (1.3) ranging between -4.4 and 0.4 , while median (IQR) of total density without head was -1.6 (1.35) ranging between -3.7 and 0.516 .

Figure 1 shows DXA scan of 2 of our patients, one with normal scan (A) and one of the cases with osteoporosis (B). The case with osteoporosis was an-11-year old girl. She has 
history of delayed motor development. She had upper limb long bone fractures twice (the first at age of 6 years, the second was 6 months later). She was not compliant to starch therapy. Her calcium, phosphorus and ALP levels were normal. She had elevated CK level (1041 U/l), deficient vitamin D2 level (17.3 ng/ml) and normal PTH level (49.6 $\mathrm{pg} / \mathrm{ml}$ ). She had widespread myopathy in EMG and mild septal hypertrophy in echocardiography. Her DXA scan results: total body density -3.2 , spine density -3.8 of the Z-score.

Cases with LBMD were compared to patients with normal BMD as regards demographic data, liver biochemical profiles, metabolic control (Table 2) as well as parameters of muscle affection, and bone metabolism (Table 3). Although patients with LBMD had lower vitamin D2 levels and higher PTH level than patients with normal BMD, it did not reach a statistically significant difference. History of fractures was more frequent in patients with LBMD (38.5\%) compared to patients with normal BMD (8.5\%) with near significant statistical difference ( $\mathrm{P}=0.07)$. Patients with LBMD significantly had elevated CK levels $(\mathrm{p}=0.04)($ Table 3$)$.

Six patients had history of fracture. All of them had elevated CK, 5 (83\%) had myopathy in EMG and 4 (67\%) had cardiomyopathy. Three patients (50\%) had hypocalcemia and low PTH, 2 (33\%) had hypophosphatemia and all of them had vitamin D2 deficiency. Figure 2 shows a Venn diagram for cases, where 23 out of the 25 children with GSD III had one or more an extra-hepatic affected organ (either cardiac, muscle or bone). Four patients (16\%) had both myopathy and LBMD, three (12\%) had both myopathy and cardiac affection, one patient had both LBMD and cardiac affection and five patients (20\%) had myopathy, LBMD and cardiac affection. 


\section{Discussion:}

Patients with GSD III have significantly abnormal bone mass, with increased risk of potential fracture. Seven of fifteen (47\%) GSD III patients (age ranging from 10 years to 34 years) were reported to have a positive history of fracture by Mundy et al. [15]. Six $(24 \%)$ of our patients had history of fractures and the median age (IQR) at first fracture was 7 (7) years ranging between 2 and 14 years. Our results are similar to that of Sentner et al. in [8] who found bone fractures in 16 GSD III patients with median age 7.5 years (range 1-18), suggesting the early development of reduced BMD in children with GSD III. Pathophysiology of reduced BMD in patients with GSD III is unclear, but an association with specific nutritional deficiencies and reduced metabolic control may have a role [1].

DXA scan in the present study demonstrated that 52\% had LBMD, two of them had frank osteoporosis. Median (IQR) of density at the spine was -1.90 (1.3) ranging between -4.4 and 0.4 , while median (IQR) of total density without head was -1.6 (1.35) ranging between -3.7 and 0.516. This finding is similar to Melis et al. [1] who studied nine GSD III patients (age 2- 20 years) and compared them to eighteen age and sex matched controls and found that GSD III patients showed lower Z-score than controls with DXA scan $(-1.21 \pm 0.74$ vs $0.74 \pm 0.14, p=0.05)$. This was also similar to Mundy et al. [15] who studied 15 GSD III patients from childhood to adulthood and demonstrated that the mean \pm SD BMD scores for all sites were significantly less than zero (whole body SD 1.47 (1.2), $\mathrm{p}<0.001$; lumbar spine SD -2.1 (1.3), $\mathrm{p}<0.001$; hip SD -1.2 (0.6), $\mathrm{p}<0.001)$ and the proportion of patients with SD scores below -2 was $40 \%$ for whole body, $64 \%$ for lumbar spine and $11 \%$ for hip. 
In patients with GSD, cross-sectional studies have demonstrated a reduction in their BMD placing them at increased risk of potential fracture. These changes have been varyingly attributed to dietary deficiencies of calcium and vitamin D2 [15]. In our study although patients with LBMD had lower vitamin D2 levels than cases with normal BMD but it did not reach statistically significant difference. Moreover, vitamin D2 was decreased on both cases and controls without a statistically significant difference. This is similar to Mundy et al. [15] who found no differences in plasma 25-hydroxyvitamin D between cases with GSD III and controls.

PTH and vitamin D are two major regulators of mineral metabolism. They play critical roles in the maintenance of calcium and phosphate homeostasis as well as the development and maintenance of bone health. PTH and Vitamin D form a tightly controlled feedback cycle, PTH being a major stimulator of vitamin D synthesis in the kidney while vitamin D exerts negative feedback on PTH secretion (16). In the current study cases with vitamin D2 deficiency significantly had higher PTH levels $(\mathrm{P}=0.001)$ and cases with high PTH levels significantly had lower calcium $(\mathrm{P}=0.05)$ and higher ALP levels $(\mathrm{P}=0.04)$ than cases with normal PTH levels.

In the present study hypocalcemia was present in about half of the patients. This hypocalcemia may be secondary to the intake of starch-based diet that may reduce the intake of micronutrients [8]. Although hypocalcemia was present more frequently in our patients with LBMD, the difference was not statistically significant $(p=0.6)$. Accordingly, calcium supplementation for these patients, particularly during the rapid childhood growth phases is recommended as suboptimal calcium intake in childhood may 
predict decreased peak bone mass and is a risk factor for adult osteoporosis and fracture [17].

Ketotic hypoglycemia is one of the hallmarks of GSD III [18]. Ketosis may affect BMD, acting through a multifactorial mechanism [19]. Therefore suboptimal metabolic control, associated with variable grades of ketosis, may play a role in reduced BMD. High protein intake may contribute to ketosis as well [20]. For GSD III, blood or urinary ketones could be used to assess optimal metabolic control of such cases $[\mathbf{1 8}, \mathbf{2 1}]$. In the current study, there were no statistically significant differences between cases with LBMD and cases with normal BMD as regards triglycerides, lactates, RBS and compliance to starch therapy $(\mathrm{p}=0.4, \mathrm{p}=0.7, \mathrm{p}=0.3, \mathrm{p}=0.7)$ respectively. One of our study limitations is that we did not measure serum or urinary ketones as a marker of metabolic control.

Consequently, we could not assess its relation to the BMD.

Muscles play a pivotal role in normal bone development and mineralization.

Consequently, GSD III patients have lower bone mass at all skeletal sites [15]. Although myopathy was present more frequently in our patients with LBMD, yet the difference was not statistically significant and patients with high CK levels significantly had lower BMD than patients with normal CK levels $(\mathrm{P}=0.04)$.

One of the strength points in our study is that it included only pediatric GSD III patients. Second, we studied a larger number of patients than other studies. Despite our study limitations, this study provides important information regarding GSD III patients and its findings highlight areas for health care providers to focus clinical attention, assessment, and intervention when dealing with this specific group of patients. 


\section{Conclusion:}

Pediatric GSD III patients have a significant prevalence of developing LBMD. It is strongly associated with myopathic changes but not significantly related to metabolic control. 


\section{References:}

1- Melis D, Rossi A, Pivonello R, et al. Reduced bone mineral density in glycogen storage disease type III: evidence for a possible connection between metabolic imbalance and bone homeostasis. Bone 2016; 86: 79-85.

2- Svedbom A, Hernlund E, Ivergård M, et al.; EU Review Panel of IOF. Osteoporosis in the European Union: a compendium of country-specific reports. Arch Osteoporos 2013; 8: 137.

3- Bethel M, Carbone L, Lohr K, Machua W. Osteoporosis. Medscape, drugs and diseases. Available at: http://emedicine.medscape.com/article/330598-overview. Updated: May, 2017. Accessed: December 2020.

4- Sanchez AJ, Aranda-Michel J. Liver disease and osteoporosis. Nutr Clin Pract 2006; 21: 273-278.

5- Lorentzon M, Cummings SR. Osteoporosis: the evolution of a diagnosis. J Intern Med 2015; 277: 650-61.

6- Mantravadi M, Woerner S, DiMeglio L. Pediatric osteoporosis. Medscape, drugs and diseases, pediatrics. Available at: http:// emedicine. medscape. com/article/985221overview. Updated: May, 2016. Accessed: December, 2020.

7- Mundy H, Lee PJ. The glycogen storage diseases. Current Pediatrics 2004; 14: 407413.

8- Sentner CP, Hoogeveen IJ, Weinstein DA, et al. Glycogen storage disease type III: diagnosis, genotype, management, clinical course and outcome. J Inherit Metab Dis 2016; 39: 697-704. 
9- Shin YS, Ungar R, Rieth M, Endres W. A simple assay for amylo-1,6-glucosidase to detect heterozygotes for glycogenosis type III in erythrocytes. Clin Chem 1984; 30: 1717-1719.

10- Shin YS, Rieth M, Tausenfreund J, Endres W. First trimester diagnosis of glycogen storage disease type II and III. J Inherit Metab Dis 1989; 12: 289-291.

11- Hollis BW, Wagner CL. Normal serum vitamin D levels. N Engl J Med 2005; 352: 515-6.

12- Kocks J, Ward K, Mughal Z, Moncayo R, Adams J, Hogler W. Z-score comparability of bone mineral density reference databases for children. J Clin Endocrinol Metab 2010; 95: 4652-4659.

13- Carter DR, Bouxsein ML, Marcus R. New approaches for interpreting projected bone densitometry data. J Bone Miner Res 1992; 7: 137-45.

14- Bachrach LK, Gordon CM. Bone densitometry in children and adolescents. Pediatrics Am Acad Pediatr 2016; 138: 4.

15- Mundy HR, Williams JE, Lee PJ, Fewtrell MS. Reduction in bone mineral density in glycogenosis type III may be due to a mixed muscle and bone deficit. J Inherit Metab Dis 2008; 31: 418-23.

16- Khundmiri SJ, Murray RD, Lederer E. PTH and vitamin D. Compr Physiol 2016; 6: $561-601$.

17- Kalkwarf HJ, Abrams SA, DiMeglio LA, Koo WW, Specker BL, Weiler H; International Society for Clinial Densitometry. Bone densitometry in infants and young children: the 2013 ISCD Pediatric Official Positions. J Clin Densitom 2014; 17: 243-57. 
18- Dagli A, Sentner CP, Weinstein DA. Glycogen storage disease type III. In: Pagon RA, Bird TD, Dolan CR, Stephens K, Adam MP, editors. GeneReviews Seattle:

University of Washington, Available from: http:// www.ncbi. nlm. nih. gov/

books/NBK26372/. Updated: 29/12/2016. Accessed: 6/10/2020.

19- Bergqvist AG, Schall JI, Stallings VA, Zemel BS. Progressive bone mineral content loss in children with intractable epilepsy treated with the ketogenic diet. Am J Clin Nutr 2008; 88: 1678-84.

20- Auvin S. Should we routinely use modified Atkins diet instead of regular ketogenic diet to treat children with epilepsy? Seizure 2012; 21: 237-40.

21- Szymańska E, Jóźwiak-Dzięcielewska DA, Gronek J, et al. Hepatic glycogen storage diseases: pathogenesis, clinical symptoms and therapeutic management. Arch Med Sci Arch Med Sci 2021; 17: 304-313. 
Figure 1: DXA scan of 2 GSD III cases of the study group

(a) Normal DXA scan (b) DXA scan with osteoporosis (total body density -3.2, spine density -3.8 of the Z-score).

DXA: Dual-emission-X-ray absorptiometry, GSD: Glycogen storage disease 
Figure 2: Venn diagram for 25 children with glycogen storage disease III (myopathy, cardiac affection and low bone mineral density). 
Table 1: Clinical data of 25 children with glycogen storage disease III of the study group

\begin{tabular}{|c|c|c|}
\hline & Number of patients & $\%$ \\
\hline \multicolumn{3}{|l|}{ Symptoms } \\
\hline Muscle weakness & 6 & 24 \\
\hline Cardiac symptoms & 0 & 0 \\
\hline \multicolumn{3}{|l|}{ Exercise: } \\
\hline - Regular & 1 & 4 \\
\hline - Irregular & 7 & 28 \\
\hline - No & 17 & 68 \\
\hline \multicolumn{3}{|l|}{ Dietary therapy: } \\
\hline - Oral uncooked cornstarch & 25 & 100 \\
\hline - High protein diet & 21 & 84 \\
\hline - Compliance & 17 & 68 \\
\hline Symptoms suggestive of hypoglycemia & 21 & 84 \\
\hline History of fractures & 6 & 24 \\
\hline \multicolumn{3}{|l|}{ Frequency of fractures per patient: } \\
\hline - Once & 4 & \\
\hline - Twice & 1 & \\
\hline - Three times & 3 & \\
\hline \multicolumn{3}{|l|}{ Clinical examination } \\
\hline Hepatomegaly & 18 & 72 \\
\hline \multicolumn{3}{|l|}{ Neurological examination: } \\
\hline - Hypotonia & 0 & 0 \\
\hline - Hyporeflexia & 0 & 0 \\
\hline - Decreased muscle power & 2 & 8 \\
\hline Cardiac abnormalities & 0 & 0 \\
\hline
\end{tabular}


Table 2: Demographic data, liver functions and parameters of metabolic control in GSD III cases with low and normal BMD

\begin{tabular}{|c|c|c|c|c|}
\hline Parameter & $\begin{array}{l}\text { All cases } \\
(n=25)\end{array}$ & $\begin{array}{c}\text { Cases with } \\
\text { low BMD } \\
(n=13)\end{array}$ & $\begin{array}{c}\text { Cases with } \\
\text { normal } \\
\text { BMD } \\
(n=12)\end{array}$ & $\begin{array}{c}P \\
\text { value }\end{array}$ \\
\hline \multicolumn{5}{|l|}{ Demographic data } \\
\hline Age in years; mean $\pm \mathrm{SD}$ & $10.52 \pm 3.1$ & $11.4 \pm 3.4$ & $9.6 \pm 2.7$ & 0.2 \\
\hline $\begin{array}{l}\text { Sex: Male; N. }(\%) \\
\text { Female; N. }(\%)\end{array}$ & $\begin{array}{l}14(56) \\
11(44)\end{array}$ & $\begin{array}{l}6(46) \\
7(54)\end{array}$ & $\begin{array}{l}8(67) \\
4(33)\end{array}$ & 0.4 \\
\hline \multicolumn{5}{|l|}{ Liver biochemical profile } \\
\hline ALT; median (IQR) (up to $40 \mathrm{U} / 1$ ) & $176.8(178.5)$ & $119(153)$ & $176(310)$ & 0.6 \\
\hline AST; median (IQR) (up to $38 \mathrm{U} / \mathrm{l}$ ) & $182(260.5)$ & $119(181)$ & $200(282)$ & 0.6 \\
\hline Albumin; mean \pm SD (up to $3.5-5 \mathrm{mg} / \mathrm{dl}$ ) & $4.112 \pm 0.47$ & $4.1 \pm 0.5$ & $4.1 \pm 0.4$ & 0.8 \\
\hline $\mathrm{INR} ;$ mean $\pm \mathrm{SD}$ & $1.07 \pm 0.06$ & $1.1 \pm 0.1$ & $1 \pm 0.1$ & 0.5 \\
\hline \multicolumn{5}{|l|}{ Parameters of metabolic control } \\
\hline Triglycerides; median (IQR) (30-150 mg/dl) & $141(85.5)$ & $133(58)$ & $158(111)$ & 0.4 \\
\hline Lactate; median (IQR) $(<2.9 \mathrm{mmol} / \mathrm{l})$ & $2.2(3.6)$ & $2.2(3.6)$ & $1.9(3.6)$ & 0.7 \\
\hline RBS; mean \pm SD $(70-180$ mg/dl) & $96.7 \pm 23.5$ & $92 \pm 18$ & $102 \pm 28$ & 0.3 \\
\hline Patients compliant to starch therapy; N. (\%) & $17(68)$ & $8(61.5)$ & $9(75)$ & 0.7 \\
\hline
\end{tabular}

ALT: alanine transaminase; AST: aspartate transaminase; BMD: bone mineral density; GSD: glycogen storage disease; INR: international normalized ratio; RBS: random blood sugar SD: standard deviation. 
Table 3: Parameters of muscle affection and bone metabolism in GSD III cases with low and normal BMD

\begin{tabular}{|c|c|c|c|c|}
\hline Parameter & $\begin{array}{c}\text { All cases } \\
(\mathrm{n}=\mathbf{2 5})\end{array}$ & $\begin{array}{c}\text { Cases with } \\
\text { low BMD } \\
(n=13)\end{array}$ & $\begin{array}{c}\text { Cases with } \\
\text { normal } \\
\text { BMD } \\
(n=12)\end{array}$ & $\begin{array}{c}P \\
\text { value }\end{array}$ \\
\hline \multicolumn{5}{|l|}{ Parameters of muscles affection } \\
\hline CK; median (IQR) (normal $\leq 145 \mathrm{U} / \mathrm{l})$ & $728(1154.5)$ & 853 (995) & $521(1438)$ & 0.3 \\
\hline Patients with elevated CK; N. (\%) & $21(84)$ & $13(100)$ & $8(67)$ & $0.04 *$ \\
\hline Myopathy in EMG; N. (\%) & $14(56)$ & $9(69)$ & $5(42)$ & 0.2 \\
\hline Cardiomyopathy; N. (\%) & $14(56)$ & $6(46)$ & $8(67)$ & 0.4 \\
\hline \multicolumn{5}{|l|}{ Parameters of bone metabolism } \\
\hline $\begin{array}{l}\text { Calcium; median (IQR) or mean } \pm \mathrm{SD}(8.8-10.8 \\
\mathrm{mg} / \mathrm{dl})\end{array}$ & $8.92 \pm 0.82$ & $8.8(1)$ & $8.9(0.6)$ & 0.7 \\
\hline Phosphorus; median (IQR) or mean $\pm \mathrm{SD}(4-7 \mathrm{mg} / \mathrm{dl})$ & $4.70 \pm 0.78$ & $4.7(0.9)$ & $4.7(0.5)$ & 0.9 \\
\hline $\mathrm{ALP} ;$ median (IQR) or mean $\pm \mathrm{SD}$ (up to $640 \mathrm{U} / \mathrm{l}$ ) & $309 \pm 122$ & $334(162)$ & $383(47)$ & 0.3 \\
\hline PTH; median (IQR) (15-57 pg/ml) & $56.4(69.05)$ & $83(109)$ & $53(39)$ & 0.2 \\
\hline Vitamin D2; median (IQR) (>30 ng/ml) & $10.8(8.1)$ & $8.8(12)$ & $12(8.6)$ & 0.5 \\
\hline
\end{tabular}

*P< 0.05 is significant; BMD: bone mineral density; CK: creatine kinase; EMG: electromyogram; GSD: glycogen storage disease; IQR: inter-quartile range; PTH: parathormone; SD: standard deviation. 

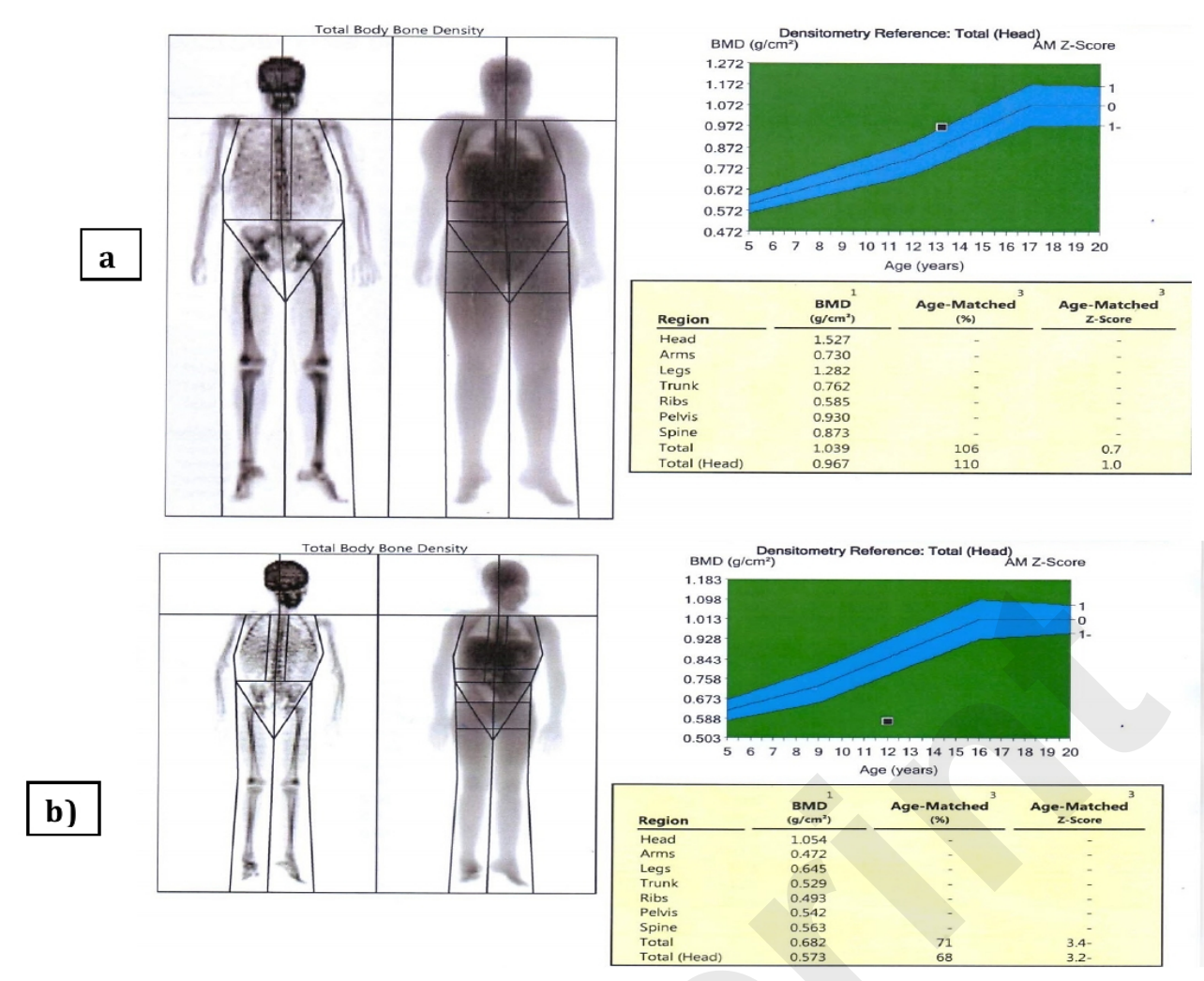

1

Figure 1: DXA scan of 2 GSD III cases of the study group 


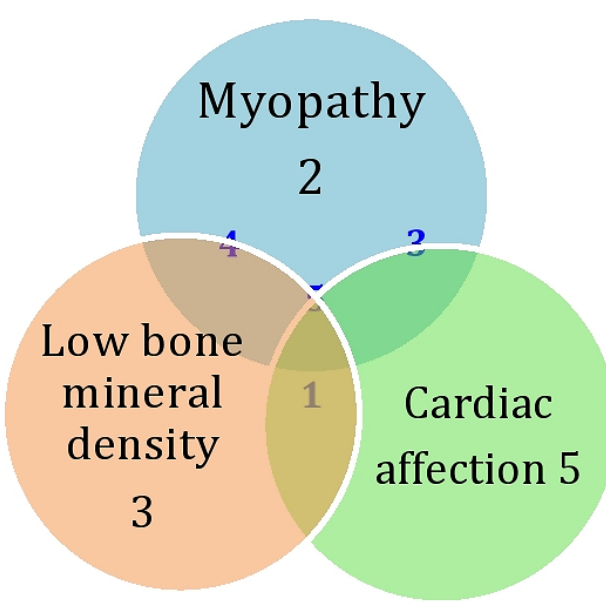

1

Venn diagram for 25 children with glycogen storage disease III (myopathy, cardiac affection and low bone mineral density) 